\title{
Estudio de caso: implementación de un modelo estratégico de comunicación organizacional
}

Estudo de caso: aplicação de um modelo estratégico de comunicação organizacional

Case study: implementation of a strategic organizational communication model 


\section{Resumen}

La metamorfosis contemporánea exige gestionar estratégicamente la comunicación organizacional e identificar las representaciones y mentalidades de los sujetos en el plano discursivo y cómo se materializan en los procesos comunicacionales. Este artículo se propone interrogar las formas de constitución de algunas mentalidades contemporáneas para plantear la necesidad de una lectura interdisciplinaria por parte del comunicador, además toma como estudio de caso Colegiatura Colombiana para plantear un modelo de comunicación estratégica de coherencia ante un mundo en metamorfosis.

\section{PALABRAS CLAVE: COSMOPOLIÉTICA•ESTUDIO DE CASO•COMUNICACIÓN ESTRATÉGICA•ESTRUCTURA ORGANIZACIONAL.}

\section{Resumo}

A metamorfose contemporânea requer uma gestão estratégica da comunicação organizacional, bem como a identificação das representações e mentalidades dos sujeitos no plano discursivo e como estas são materializadas nos processos comunicacionais. Este artigo busca questionar as formas de constituição de algumas mentalidades contemporâneas para mostrar a necessidade de uma leitura interdisciplinar do comunicador, tomando como estudo de caso a instituição Colegiatura Colombiana para propor um modelo coerente de comunicação estratégica diante de um mundo em metamorfose.

\section{PALAVRAS-CHAVE: COSMOPOLIÉTICA・ESTUDO DE CASO・COMUNICAÇÃO ESTRATÉGICA・ESTRUTURA ORGANIZACIONAL.}

\section{Abstract}

The contemporary metamorphosis requires a strategic management of organizational communication and the identification of subject's representations and mentalities in the discursive level and the way in which they materialize in the communicational processes. This article seeks to question the ways some contemporary mentalities are formed to show the need for an interdisciplinary assessment of the communicator, taking as a case study the institution Colegiatura Colombiana to propose a coherent model of strategic communication in the face of a metamorphosing world. 


\section{INTRODUCCIÓN}

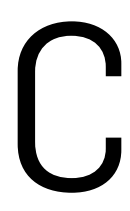

omo señaló el sociólogo alemán Ulrich Beck (2017), el mundo contemporáneo se encuentra ante un proceso de metamorfosis, un cambio profundo en la forma cómo las organizaciones, las personas, los Estados y la sociedad en general se relacionan, construyen sus mentalidades y representan el mundo.

La metamorfosis implica una transformación mucho más radical, mediante la cual las viejas certezas de la sociedad moderna se desvanecen mientras surge algo completamente nuevo. Para comprender esta metamorfosis del mundo hay que explorar los nuevos comienzos, centrándose en lo que surge de lo viejo e intentando comprender las futuras normas y estructuras que caracterizan la confusión del presente. (Beck, 2017, p.17)

La metamorfosis planteada por Beck (2017) encuentra como protagónico el papel de la comunicación como un elemento transversal y constitutivo, así como un factor dinamizador de los procesos de transformación profunda en el ámbito relacional con capacidad de sociogénesis (Martín Serrano, 2012). Ante lo anterior, la constitución epistemológica de la comunicación como disciplina ha sido completamente interdisciplinaria, lo que no debería dejar de ser una ruta integrada a la gestión de la comunicación organizacional pese a la madurez y desarrollo de la comunicación como campo de conocimiento.

Las organizaciones contemporáneas están en entornos de cambio, dinamismo, capitalismo cognitivo e hiperinformación. Desde dicho contexto requieren una comunicación estratégica empática, relacional y que tenga como referente el cuidado del lazo social, y de esto emergen tres formas de comprensión del mundo: cuidado de sí, cuidado del otro y cuidado del mundo (Campillo, 2018), las cuales son determinantes para la adecuada planeación de una comunicación estratégica y para la conexión con sus grupos de interés.

Teniendo en cuenta esto, esta investigación tomó como objeto de estudio la gestión de la comunicación organizacional de la institución de educación superior, Colegiatura Colombiana. La pertinencia de tal organización se justifica en su propósito de educación superior, su filosofía organizacional, así como sus procesos de formación y direccionamiento estratégico que se basan en lo que se ha denominado la comprensión "cosmopoliética" del mundo' (Campillo, 2018). Esta institución intenta desde la comunicación esencial² entrar en sintonía con la mentalidad de ese sujeto contemporáneo que es más líquido, móvil, cambiante y altamente volátil, pero también creativo, innovador, talentoso y consciente de sí.

El otro factor preponderante para la elección de la organización tiene que ver con lo que Colegiatura Colombiana denomina su pensamiento institucional, el cual refleja discursivamente buena parte de las tendencias organizacionales de la sociedad contemporánea que propenden por el bienestar interno del sujeto, por su integralidad física, emocional y relacional, por su coherencia y por su acción transformadora consciente individual y social.

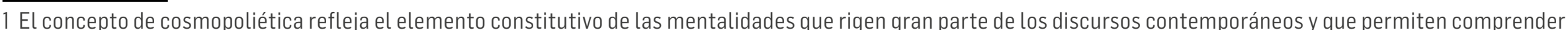

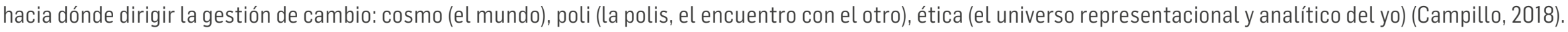

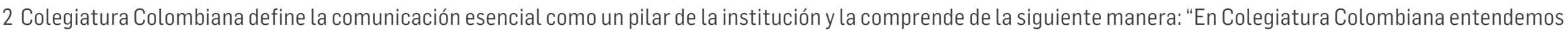

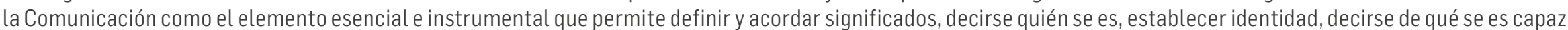

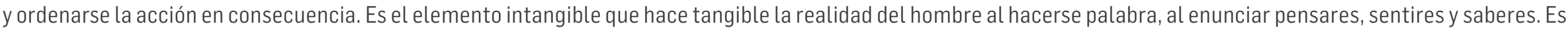

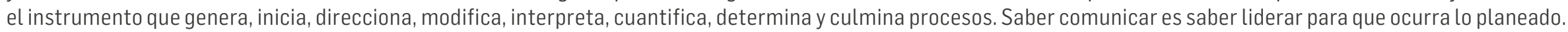

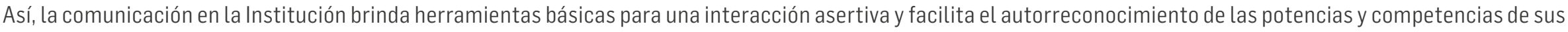
integrantes" (Colegiatura Colombiana, 2019).
} 
Así, este caso de estudio se centró en el proceso comunicacional de Colegiatura Colombiana desde su campo discursivo, pero también se exploró un modelo que podría articularse con sus principios de enunciación, con el fin de saber qué tipo de comunicación se debe liderar en una organización de este tipo y lograr, desde la gestión de la comunicación organizacional, una articulación y proyección en sus públicos interno y externo. Además, se propuso un modelo estructural para su sistema de comunicaciones que logre dar cuenta del pensamiento institucional y de sus modos de comprensión del mundo.

\section{METODOLOGÍA}

Este trabajo utilizó un modelo que, según la exploración realizada en la fase de formulación del proyecto, permite materializar y articular los sistemas de comunicación organizacional con la forma cómo las organizaciones en la contemporaneidad demandan mayor alcance y compresión estratégica de su quehacer misional. Tal modelo se estructura bajo la siguiente serie lógica: políticas, procesos, estructura, recursos y estrategia (Aristizábal Giraldo; Hernández García, 2012). La aplicación de dicho modelo permitirá definir, por ejemplo, la planeación estratégica del cambio organizacional (Kotter, 2005), la gestión de intangibles o la gestión de la cultura en las organizaciones de acuerdo con la mentalidad y paradigmas de los sujetos que las habitan.

De la metodología utilizada se tomó un estudio de caso para rastrear los elementos que subyacen a la problemática planteada. Desde allí se buscó caracterizar el alcance de las nuevas formas de expresión organizacional a partir de un modelo de comunicación estratégica para proyectar una identidad organizacional con un tono contemporáneo.

La estrategia metodológica respondió a una investigación cualitativa, de carácter descriptivo y que se configuró a partir de dos momentos: en el primer se realizó una revisión bibliográfica para atender a la necesidad de fundamentar teóricamente la relación de la cosmopoliética contemporánea (cuidado de sí, del otro y del mundo) con los conceptos subyacentes al pensamiento institucional de Colegiatura Colombiana, que se manifiestan en un abanico de autores en su mayoría provenientes de la sociología y la filosofía contemporáneas.

Es desde estos campos del saber que la comunicación organizacional debe comprender la mediación social del sujeto al que se dirige y con el cual cocrea. Así mismo se revisó toda la documentación en la que se ilustran y enuncian los fundamentos del pensamiento institucional de Colegiatura, sin encontrar ningún documento de corte académico más si documentación de carácter administrativo para explicar su filosofía organizacional.

Para sustentar la perspectiva conceptual de la cosmopoliética y su relación con los conceptos constitutivos del pensamiento institucional, fue necesario acercarse a la denominada metamorfosis de la experiencia existencial del sujeto y su lazo social. Dicha metamorfosis constituye el interés central de las investigaciones que han orientado el presente proyecto, en tanto se han encargado de cuestionar e indagar las dinámicas sociales en las que la comunicación ha encontrado un nicho para su desarrollo.

El segundo momento surgió posterior a la exploración bibliográfica y de los referentes organizacionales reconocidos. En este se establecieron categorías de materialización desde un modelo de comunicación estratégica, categoría que se describen en este apartado metodológico. Una vez identificadas se establecieron las relaciones con los factores constitutivos del pensamiento institucional de Colegiatura Colombiana.

Tal momento de la estrategia metodológica permitió realizar un contraste del marco teórico abordado con el pensamiento institucional de Colegiatura como estudio de caso, en el que una organización que desde su concepción filosófica proyecta una consonancia con la cosmopoliética contemporánea. En tal sentido se exploró cómo dicho proceso se podría expresar de forma pertinente con el modelo de comunicación propuesto. 
El contraste realizado se sirvió de una perspectiva interpretativista; tal enfoque permitió comprender un estudio de caso que apela a la articulación entre la filosofía organizacional con un enfoque contemporáneo con un modelo de comunicación. El enfoque interpretativista se orientó con los siguientes objetivos, de acuerdo con Fajardo y Nivia (2016, p.116):

- Evaluar el papel de la comunicación en la creación, mantenimiento y desarrollo de la cultura de una organización.

- Evaluar el significado y contenido de las producciones comunicacionales.

- Evaluar los procesos de creación y desarrollo de las producciones comunicacionales.

- Entender la vida organizacional y el papel de la comunicación desde la perspectiva de los miembros de la organización.

El énfasis de la perspectiva interpretativista está más en el entender las prácticas de comunicación de una organización que en cambiarlas.

De otra parte, para el abordaje del estudio caso de Colegiatura Colombiana, se realizó una observación directa, en tanto

permite [que] se familiarice con la vida organizacional, su gente, su ambiente físico, y con lo que son las prácticas de comunicación tal como acontecen en sus diferentes niveles. Esta técnica permite que recoja información detallada y de primera mano sobre procesos de comunicación que son de capital importancia para la organización. (Fajardo; Nivia, 2016, p.121)

En el desarrollo del segundo momento metodológico también se realizaron talleres con la comunidad, con la cual se indagó por los cinco factores constitutivos del sistema de comunicaciones propuesto, por su percepción, conocimiento y experiencias como grupos de interés de la institución. En estas comunidades se contó con estudiantes, docentes, egresados y personal administrativo. Los resultados de los talleres permitieron identificar la sintonía entre los factores del sistema de comunicaciones con los pilares del pensamiento institucional de Colegiatura, explorar escenarios de aplicación, encontrar necesidades no atendidas pero prioritarias, visualizar acciones institucionales de alto impacto comunicacional y delinear los procesos relacionados con la gestión estratégica de la comunicación organizacional como forma de materializar y posicionar el pensamiento institucional con sus grupos de interés.

Para realizar un contraste de la información obtenida en la revisión documental y en los talleres, se realizaron entrevistas con directivos y líderes de opinión de la institución, las cuales contemplaron algunos de los elementos o factores hallados en los talleres. En las entrevistas de mayor relevancia se destacan las realizadas a tres actores claves por sus responsabilidades, roles e indecencia en las políticas institucionales, por su experticia, conocimiento y experiencia en asuntos de planeación, diseño y auditoría de procesos, de liderazgo y gestión organizacional. Se trató de entrevistas a profundidad con el líder principal de la institución, el rector de Colegiatura, con la encargada de planeación y de los procesos de aseguramiento de la calidad y con el Coordinador del Laboratorio de Creatividad Prospectiva de la institución. Las entrevistas permitieron identificar la pertinencia del modelo propuesto con base en los cinco factores del sistema de comunicaciones y la necesidad de pensarlo desde una perspectiva de comunicación estratégica.

Tanto la exploración documental como los métodos utilizados requirieron una guía para estructurar el proceso de objetivación e indagación; desde allí el trabajo investigativo se fundamentó en el modelo propuesto por Aristizábal Giraldo y Hernández García (2014, p.101): "Los lineamientos propuestos para construir el modelo de comunicación se orientan a los cinco factores determinados para la evaluación de las instituciones: las políticas, los procesos, la estructura, los recursos y la estrategia". Un modelo que permitió la revisión del sistema de comunicación de Colegiatura y la forma cómo desde allí es posible proyectar la gestión estratégica de su identidad organizacional bajo la cosmopoliética.

Los factores utilizados en la estrategia metodológica y algunos de los elementos de articulación con el estudio de caso de Colegiatura Colombiana, se enuncian a continuación. 


\section{Políticas}

La política de comunicación es la guía para saber cómo se debe actuar, el norte de la institución en cuanto al consenso sobre lo que se comprende por comunicación, el grado de interacciones y el alcance de la misma, y expresa su esencia como proceso comunicacional.

El punto clave de la política de comunicación de toda organización es su apropiación, observancia y efectividad. Esta se mide con preguntas como: ¿Qué tan útil es? ¿se comprende como propia la política de comunicación interna en la institución? ¿es clara y práctica para todos los grupos de relación? Estos cuestionamientos permiten aclarar el panorama de la comunicación, por lo tanto, su análisis riguroso y detallado ayudará a construir la discusión que lleve a definir la política.

\section{Procesos}

En primera instancia es fundamental comprender lo que implica el proceso como condición de posibilidad de la gestión de la comunicación interna, como fuente y realidad. Un proceso es "un conjunto de actividades de trabajo interrelacionadas, que se caracterizan por requerir ciertos insumos (inputs: productos o servicios obtenidos de otros proveedores) y actividades específicas que implican agregar valor, para obtener ciertos resultados (outputs)" (Mallar, 2010, p.7).

La estructura de gestión por procesos de la comunicación implica comprenderla como un asunto sistémico, que supera la transmisión de información y se orienta a la creación de sentido desde la generación de relaciones de valor o vínculos estratégicos tanto internos como externos. Así comprende Colegiatura la gestión por procesos de la comunicación organizacional, algo que se identificó en las entrevistas como una forma de gestión a la que se le apuesta y, por tanto, el modelo de comunicación propuesto se articula con su identidad organizacional.

\section{Estructura}

Cuando se aborda la estructura se hace referencia al nivel estratégico en el que, desde la arquitectura organizacional, se va a adscribir el área responsable de la comunicación, así mismo se debe considerar su participación en mecanismos laterales, como comités o comisiones, que orienten el desarrollo de procesos y proyectos institucionales, para en todo caso estar ligado al espíritu y a la vida de la institución.

La estructura responsable de la gestión estratégica de la comunicación debe velar por su participación e interlocución en las instancias decisorias, de planeación, proyección y prospectiva de la organización. De acuerdo con lo anterior es fundamental comprender que la estructura organizacional de la institución deberá incorporar en los diversos procesos misionales la asesoría y el acompañamiento de los responsables de la comunicación.

\section{Recursos}

Frente a los recursos se estima que el más importante es el talento humano con experiencia, experticia, criterio y capacidad de asesorar estratégicamente a la organización en asuntos de gestión de cambio, cultura, clima, conocimiento, riesgos y crisis. De lo cual se desprende que es importante que el equipo responsable de la comunicación también cuente con conocimiento del sector educación, capital relacional y apropiación de la cultura desde lo discursivo, las relaciones de poder y las mejores formas para crear, apostar y resignificar acciones. 
Los recursos para la gestión de la comunicación deben asegurar un área de comunicaciones que se encargue de los procesos, disponer del personal competente para desarrollar los programas, los medios y las acciones que posibiliten el logro de los objetivos de comunicación. (Andrade, 2005, p.67)

Los recursos destinados a la gestión de las comunicaciones son en buena medida señal del alcance y capacidad de respuesta de la organización frente a las necesidades.

\section{Estrategia}

Si se parte de la definición de "estrategia" se podría comprender gran parte del alcance de la misma: es un plan para dirigir un asunto; se compone de una serie de acciones planificadas para conseguir resultados más efectivos. Una serie de prácticas que son medidas concretas para alcanzar uno o varios objetivos (Estrategia..., 2014). Tales medidas concretas deben responder a una estructura que haga posible lo que se ha propuesto.

Por su parte, las expertas en comunicación estratégica Ángela Preciado y Haydée Hoyos (2012, p.130) señalan que "la estrategia se hace presente en la política, en las empresas, en los deportes, en el ámbito social, y también se aplica en situaciones en las que las personas se organizan para lograr un propósito". Por esto, el factor estrategia es entendido desde la planeación y la forma como aporta al cumplimiento de la misión y la visión de las organizaciones, pues lleva a que los grupos de interés internos participen de forma activa en el diseño de la estrategia global permitiendo del diálogo, la evaluación y el seguimiento de las diferentes acciones internas y externas de la organización.

Por medio de las estrategias se define el norte o la guía para encaminar los objetivos al cumplimiento exitoso no solo del marco estratégico organizacional, sino también su propósito fundamental.

De esta manera, las estrategias planeadas desde la comunicación permiten la visión holística del entorno, buscan el consenso de los diferentes públicos de interés, convergen los objetivos de los diferentes equipos para así articular tiempos, detectar las necesidades y recursos necesarios.

\section{Referentes teóricos}

Como se ha logrado evidenciar el principal referente teórico de la investigación proviene de la comunicación estratégica, con los aportes de Aristizábal Giraldo y Hernández García (2014) con el modelo propuesto, pero también de Preciado y Hoyos (2012). En el presente apartado se hará mayor énfasis al componente interdisciplinario que orientó la investigación y de su importancia para que discursivamente el modelo propuesto logre conversar con la perspectiva cosmopoliética contemporánea que orienta el pensamiento institucional de Colegiatura Colombiana.

La convergencia entre el mundo contemporáneo con todos sus condicionantes, el individuo con su psique y el universo de apropiación sociocultural de la comunicación, determinan la existencia y la dinámica de relación entre instituciones, sujetos y discursos. El mundo de la segunda mitad del siglo XX nos ha tenido como testigos de una metamorfosis colectiva que experimenta la sociedad y el individuo de la contemporaneidad; no solo es una transformación, un cambio o una revolución; en este punto se converge con Beck (2017) en que se experimenta una profunda metamorfosis de las creencias, representaciones, lenguajes y, en especial, de la vida material. Esto último está evidenciado en la perspectiva postindustrial 4.0 y en la proyección biotecnológica del transhumanismo y la singularidad, es decir, desde lo 5.0. 
La hegemonía de la globalización, el gobierno del mercado, el control expansivo de la tecnología y la imperante individualización del mundo contemporáneo se constituyen en cuatro factores que disuelven las pocas certezas que había dejado el discurso postmoderno (Lipovetsky, 2008). Son a la vez procesos que configuran al sujeto neoliberal de la segunda década del siglo XXI (Laval; Dardot, 2013), en el que la comunicación digital (Scolari, 2008), la autogestión (Han, 2014), ladividualidad (Deleuze, 2006) y la ubicuidad (López-Portillo, 2018) propias del capitalismo informacional determinan la relación entre instituciones, sujetos y discursos. De esta manera, "el mundo está experimentando una sorprendente pero comprensible metamorfosis mediante la transformación del horizonte referencial y de las coordinadas de acción, que tácitamente se consideran constantes e inmutables" (Beck, 2017, p.30).

En la sociedad contemporánea se configuran nuevos relatos en los que el ser humano parece estar encontrando un salvavidas sobre el cual aferrarse en el mundo líquido que habita. Esto es lo que Antonio Campillo (2018) denomina la "cosmopoliética", un reflejo de representaciones mentales cambiantes y de paradigmas contemporáneos que reivindican campos de saber y experiencias de vida para fundamentar objetos de estudio coherentes con el ethos del sujeto contemporáneo, un ethos cosmopoliético. De esta manera,

\footnotetext{
Los paradigmas que sostienen dicho ethos, ámbitos como el cuidado de sí (alimentación consciente, estilos de vida saludable y autorreconimiento del potencial ilimitado propio), el cuidado del otro (reconocimiento de las diferencias, derechos igualitarios, inclusión y participación plena) y el cuidado del entorno (protección del medio ambiente, cuidado y militancia por los derechos de los animales, promoción de energías limpias), ámbitos potenciados por las conexiones globales, por los puentes construidos con la comunicación digital y cuyos contenidos se expanden en la medida en que dicha comunicación permea todas las capas sociales y las del individuo mismo. (Castro, 2021, p.30)
}

Ahora bien, los discursos generados en el marco de la metamorfosis del mundo contemporáneo encuentran en dicho ethos cosmopoliético referentes orientadores para la gestión comunicacional y para la generación de contenidos de todo tipo, con enfoque centrado en especial en el cuidado de sí, un enfoque en el trabajo del sujeto consigo mismo. Desde allí emerge el concepto de "consciencia" de sí, el cual puede tener una definición que parta del sentido común, como la acción de ser consciente del entorno y de las acciones propias, el darse cuenta de, siendo así incluso se podría recurrir a la definición del diccionario de la RAE que presenta cuatro acepciones:

\footnotetext{
1. f. Capacidad del ser humano de reconocer la realidad circundante y de relacionarse con ella. El coma consiste en la pérdida total de la consciencia. 2. f. Conocimiento inmediato o espontáneo que el sujeto tiene de sí mismo, de sus actos y reflexiones. Perdió la consciencia de lo que le estaba pasando.
}

3. f. Conocimiento reflexivo de las cosas. Actuó con plena consciencia de lo que hacía.

4. f. Psicol. Acto psíquico por el que un sujeto se percibe a sí mismo en el mundo. (Consciencia, 2021)

El abordaje de la consciencia de sí desde la acción de las psicociencias en la configuración del mundo contemporáneo (Bedoya Hernández, 2018) permite abrir otra perspectiva desde la mirada de las tecnologías del yo desarrollada por Michel Foucault (2015) y Gilles Deleuze. Desde allí adquiere relevancia la mirada crítica de lo que implica la racionalidad neoliberal para la compresión del sujeto sobre sí mismo, como bien lo plantean Alain Ehrenberg (2014), Byung-Chul Han (2014), Eva Illouz (2019), Christian Laval y Pierre Dardot (2013) y Richard Sennett (2000), que muestran los efectos de la instrumentalización de la consciencia de sí como parte de la racionalidad neoliberal que profundiza la ruptura del lazo social y patologías como la depresión y el burnout.

La perspectiva de trabajo desde la conciencia de sí le da paso al resto de la serie lógica cosmopoliética: cuidado del otro y del mundo, en tanto como lo señala el pensamiento institucional de Colegiatura Colombiana: "La transformación siempre debe 
venir primero desde adentro, de uno mismo, de lo contrario no es posible transformar nada, no al menos de forma consciente y sostenible" (información verbal) ${ }^{3}$.

Desde el enfoque de la comunicación organizacional, fue necesario articular a la perspectiva de la cosmopoliética contemporánea el concepto de mediación y los múltiples factores que lo integran en lo social, lo simbólico y lo material. En tal sentido el aporte de Jesús Martín Barbero con el concepto de mediaciones fue fundamental:

\begin{abstract}
La propuesta de una teoría social de la comunicación basada en el paradigma de la mediación. Que es aquel modelo 'que trabaja con intercambios entre entidades, materiales, inmateriales y accionales' adecuado para 'estudiar aquellas prácticas en las que la conciencia, la conducta y los bienes entran en proceso de interdependencia' [...]. Un modelo que referido al campo del que nos ocupamos busca dar cuenta de las formas/instituciones que toma la comunicación en cada formación social, de las lógicas que rigen los modos de mediación entre el ámbito de los recursos. La organización del trabajo y la orientación política de la comunicación, y por último de los usos sociales de los productos comunicativos. (Martín Barbero, 2012, p.9)
\end{abstract}

Para el caso de Colegiatura Colombiana, la configuración de su pensamiento institucional como marco filosófico organizacional fue fundamental para la comprensión de los referentes teóricos identificados como una forma de comprensión interdisciplinar para formular una estrategia de comunicación pertinente y relevante para la institución.

\title{
RESULTADOS
}

En el caso del objeto de la investigación, se indagó qué tipo de sistema de comunicaciones permite sustentar la plataforma filosófica de Colegiatura Colombiana con el panorama antes esbozado de la perspectiva cosmopoliética contemporánea (cuidado de sí, del otro y del mundo) y los conceptos que subyacen al pensamiento institucional de Colegiatura. En este sentido, podemos observar que

el ethos del individuo contemporáneo trae consigo nuevos panoramas para abordar, desde el cuidado de sí, del otro y del planeta, paradigmas actualizados al mundo que sobrevivió a la posmodernidad y que requiere de nuevos relatos globales para generar el piso sobre el cual se fundamente la fe del individuo del presente y del futuro. (Castro, 2020, p.31)

Para las organizaciones se hace fundamental generar ese piso común, que responda a la comunicación como un activo estratégico de alcance social e individual y que dinamice la construcción de sentido en la mente de los sujetos respecto del lugar donde trabaja o estudia.

En este caso estudiado y teniendo en cuenta todo lo planteado anteriormente, se propuso un modelo para articularse desde una perspectiva sistémica, para dar cuenta y ser coherente con el propósito superior de la organización, con el ethos del sujeto contemporáneo y con el marco teórico identificado en la metamorfosis del mundo contemporáneo.

Los hallazgos permitieron indagar desde los elementos constitutivos de un sistema de comunicación organizacional, planteados por Aristizábal Giraldo y Hernández García (2014), el marco común que se requiere para gestionar efectivamente el proceso de cambio que viven las organizaciones, en especial las instituciones de educación superior en la que se enmarca el caso estudiado.

3 Entrevista de Julio Salleg, rector de Colegiatura Colombiana. Se llevó a cabo el 07 de abril de 2020 
Como resultado del segundo momento metodológico planteado se identificó la necesidad de comprender la comunicación como un asunto más allá de lo instrumental e informacional, un concepto que se fundamenta a partir de la apropiación sociocultural del mundo contemporáneo y que implica la generación de sentido compartido.

La comunicación se nutre de las mediaciones sociales que generan sentido para el individuo y el grupo social desde las interacciones humanas, desde los propósitos comunes y desde el lazo social que se recrea y resignifica en la medida en que la tendencia evolutiva se concreta en cualquier tipo de organización humana. (Castro; Vargas, 2020, p.92)

Se identificó como un rasgo predominante en los públicos consultados su percepción de una demanda creciente de la comunicación como asunto estratégico, al que se le asignan recursos que reflejan las apuestas institucionales, del que se espera retorno de la inversión y al que se le exigen planes de comunicaciones de amplio alcance, profundidad y articulación con el quehacer misional, pasando de los listados de actividades puntuales a estrategias diseñadas para contribuir con el posicionamiento interno y externo.

Se observó que la gestión de la comunicación organizacional en Colegiatura Colombiana no se trata solo de información, sino que es un asunto del cual se espera un liderazgo con sentido, contexto y conocimiento profundo del pensamiento institucional como filosofía orientadora de la organización.

En adelante se esbozará de forma esquemática cada uno de los hallazgos respecto de los factores que constituyeron el modelo de sistema de comunicaciones que se usó como referente y horizonte metodológico, lo que podrá servir para su aplicación en la institución.

\section{Políticas}

Para el caso estudiado no solo se trata de comunicación organizacional universitaria, sino que debe comprender la filosofía institucional, desde la cual se comprende que la política responde a lo que su plataforma estratégica define como comunicación esencial. De esta manera, es desde una comprensión de la comunicación como un pilar institucional que se debe fundamentar la política de comunicaciones. Vale anotar que tal política está en construcción como una de las aplicaciones derivadas de la investigación.

A partir de una política de comunicaciones formulada en coherencia con el pensamiento institucional de Colegiatura Colombiana, que se fundamenta en la perspectiva cosmopoliética contemporánea, se vislumbra el horizonte de comprensión con el que los directivos de la institución encuentran pertinente y con amplio alcance a la gestión de la comunicación organizacional.

\section{Procesos}

De la observación e identificación en Colegiatura se encontró una necesidad que predomina en la gestión comunicacional. Se trata de la búsqueda por formalizar y sistematizar los procesos propios de la comunicación organizacional para tenerlos plenamente identificados y materializarlos en los procesos ejecutables y en las actividades de trabajo, en tanto al no tenerlos plenamente caracterizados genera reprocesos organizacionales.

Si se comprende que los procesos de comunicación se convierten en hojas de ruta, en la posibilidad de acompañar procesos, de facilitar desde el liderazgo consciente y estratégico asuntos de amplia importancia en la institución, tenerlos plenamente caracterizados permitiría desplegar la dimensión estratégica de la comunicación. 


\section{Estructura}

En este sentido, el área de comunicaciones de Colegiatura Colombiana se encuentra en la máxima instancia de direccionamiento estratégico (Rectoría) y tiene asiento en importantes instancias decisorias, lo que implica que su nivel de influencia y acompañamiento logra permear los asuntos de mayor relevancia institucional. Pese a ello, como se logró identificar en los talleres, el impacto y gestión de la comunicación organizacional en Colegiatura aún es muy instrumental y operativo por la falta de una política clara, de procesos delineados y de una estrategia orientadora. Lo anterior se encuentra en construcción como efecto derivado de la presente investigación.

\section{Recursos}

Desde esta perspectiva, la institución cuenta con un componente sólido de recursos, un equipo de profesionales con conocimiento y talento, equipos que en la medida de las necesidades responden con los contenidos generados, y en cuanto al presupuesto responde a la gestión desarrollada. Es importante anotar que la perspectiva de liderazgo del área de comunicaciones requiere una mayor visión estratégica e interdisciplinar para la comprensión y mejor utilización de los recursos con los que cuenta.

\section{Estrategia}

Colegiatura Colombiana tiene un componente estratégico fundamental y potente como es el pensamiento institucional y su orientación desde la cosmopoliética contemporánea. Lo anterior sirve como plataforma de fundamentación de la comunicación organizacional, pero desde ninguna perspectiva reemplaza un plan de comunicación estructurado con los componentes señalados anteriormente. Esta es una deficiencia identificada en el área de comunicaciones que no cuenta con un plan acorde con lo señalado en los anteriores factores ni con una estrategia gruesa para atender a las necesidades de los grupos de interés con los cuales interactúa Colegiatura Colombiana.

\section{CONSIDERACIONES FINALES}

Colegiatura Colombiana como institución de educación superior orienta su filosofía institucional hacia los factores propios de la perspectiva de la cosmopoliética contemporánea (cuidado de sí, del otro y del mundo) y encuentra en el sujeto de la actualidad un perfil apto y proclive a engancharse con estrategias comunicacionales más sensibles al diálogo y la cocreación.

El desarrollo de un sistema de comunicación organizacional sustentado en políticas, procesos, estructura, recursos y estrategia permite como modelo lograr una completa coherencia con las tendencias contemporáneas, para una gestión estratégica de la comunicación organizacional.

La constitución de un plan de comunicación estratégica desde los hallazgos identificados se hace urgente para lograr un mayor posicionamiento de la institución, una proyección de su pensamiento institucional y un mayor enganche e identificación en coherencia con el perfil del sujeto contemporáneo identificado, con sus mayores necesidades, déficits y urgencias existenciales.

El lenguaje, los públicos, las sensibilidades, así como la comunicación horizontal entre los principales grupos de interés para el caso de Colegiatura se constituyen en oportunidades para la gestión estratégica de la comunicación, se encuentran como coherentes e impulsan un enganche con su plataforma de pensamiento. 
La coherencia entre el aspecto discursivo y la implementación de las decisiones organizacionales debe ser una prioridad para la gestión estratégica de la comunicación. Esto se deriva de la forma como las personas habitan las organizaciones en el marco de sistemas de pensamiento que responden, desde su representación mental, a la perspectiva cosmopoliética contemporánea.

\section{REFERENCIAS}

ANDRADE, Horacio. Comunicación organizacional interna: proceso, disciplina y técnica. Oleiros: Netbiblio, 2005.

ARISTIZÁBAL GIRALDO, María Eugenia; HERNÁNDEZ GARCÍA, David. Estudio comparativo de la gestión de la comunicación interna en universidades públicas con acreditación de alta calidad en Antioquia. Folios, Revista de la Facultad de Comunicaciones, Medellín, n.31, p.91-106, 2014.

BECK, Ulrich. La metamorfosis del mundo. Barcelona: Paidós, 2017.

BEDOYA HERNÁNDEZ, Mauricio. La gestión de símismo. Medellín: Editorial Universidad de Antioquia, 2018.

CAMPILLO, Antonio. Mundo, nosotros, yo. Barcelona: Herder, 2018.

CASTRO, Fernando. El presente que nos habita. Medellín: Fondo Editorial Colegiatura, 2021.

CASTRO, Fernando. Tecnología, disciplina y control. In: FELTRERO, Roberto (ed.). La enseñanza de las ciencias desde la pedagogía social:el paradigma educativo STEM como modelo para la educación integral de ingenieros y ciudadanos. Madrid: GKA, 2020. p.21-32.

CASTRO, Fernando; VARGAS, Valentina. La comunicación organizacional un proceso que transforma y se transforma. In: CASTRO, Fernando; VARGAS, Valentina. Estrategias y metodologías de creación/comunicación. Medellín: Fondo Editorial Colegiatura, 2020. p.87-103.

COLEGIATURA COLOMBIANA. Pensamiento institucional. Medellín, 2019. Disponível em: https://www.colegiatura.edu.co/ colegiatura/pensamiento/\#Pilares. Acesso em: 8 out. 2021.

CONSCIENCIA. In: DICCIONARIO de la lengua española. Madrid: RAE, 2021. Disponível em: https://dle.rae.es/consciencia?m=form. Acesso em: 11 out. 2021.

DELEUZE, Gilles. Conversaciones. Valencia: Pre-textos, 2006.

EHRENBERG, Alain. De la neurosis a la depresión. París: ERES, 2001.

ESTRATEGIA. In: DICCIONARIO de la lengua española. Madrid: RAE, 2014. Disponível em: https://dle.rae.es/estrategia. Acesso em: 18 out. 2021.

FAJARDO, Gloria; NIVIA, Adriana. Relaciones públicas y comunicación organizacional: ejes de la comunicación. Bogotá: Universidad de Bogotá Jorge Tadeo Lozano, 2016. 
FOUCAULT, Michel. Tecnologías del yo. Barcelona: Paidós, 2015.

HAN, Byung-Chul. Psicopolítica. Madrid: Herder, 2014.

KOTTER, John P. Liderar el cambio en gestión del cambio. Santiago de Chile: Harvard Bussines Review; Planeta Chilena, 2005.

LAVAL, Christian; DARDOT, Pierre. La nueva razón del mundo. Barcelona: Gedisa, 2013.

LIPOVETSKY, Gilles. La era de la decepción. Barcelona: Anagrama, 2008.

LÓPEZ-PORTILLO, José Ramón. La gran transición. Ciudad de México: Fondo de Cultura Económica, 2018.

MALLAR, Miguel Ángel. La gestión por procesos: un enfoque de gestión eficiente. Revista Científica "Visión de Futuro", Miguel Lanús, v.13, n.1, 2010.

MARTÍN BARBERO, Jesús. De los medios a las mediaciones. comunicación, cultura y hegemonía. Barcelona: Anthropos, 2012.

MARTÍN SERRANO. Miguel. Avances científicos y cambios sociales que están transformando los estudios de la comunicación. In: SEMINARIO INTERNACIONAL COMUNICACIÓN, VIDA Y SOCIEDAD, 1., 2010, Medellín. Anais [...]. Medellín: Universidad de Antioquia, 2010.

PRECIADO, Ángela; HOYOS, Haydée. Usos y prácticas de la comunicación estratégica en las organizaciones. Bogotá: Universidad de la Sabana, 2012.

SCOLARI, Carlos Alberto. Hipermediaciones. Barcelona: Gedisa, 2008.

SENNETT, Richard. El respeto: sobre la dignidad del hombre en un mundo de desigualdad. Barcelona: Anagrama, 2003.

Artículo recibido el 31.05.2021 y aprobado el 27.09.2021. 